\title{
Simulation for Pre-Visualizing and Tuning Lighting Controller Behavior
}

\author{
Li Jia ${ }^{a}$, Sina Afshari ${ }^{\mathrm{a}}$, Sandipan Mishra ${ }^{\mathrm{b}}$, Richard J. Radke ${ }^{\mathrm{a}, *}$ \\ ${ }^{a}$ Department of Electrical, Computer, and Systems Engineering, Rensselaer Polytechnic \\ Institute, 110 8th Street, Troy, NY, 12180, USA \\ ${ }^{b}$ Department of Mechanical, Aerospace, and Nuclear Engineering, Rensselaer Polytechnic \\ Institute, 110 8th Street, Troy, NY, 12180, USA
}

\begin{abstract}
We present a computer graphics simulation framework to pre-visualize and tune the parameters of an advanced lighting controller for a given illuminated environment. The objective is to show that the simulation framework makes it easy for a user to predict the controller's behavior and modify it with minimal effort. Our methodology involves off-line pre-computation of lightmaps created from photorealistic rendering of the scene in several basis lighting configurations, and the subsequent combination of these lightmaps in a video game engine. We demonstrate our framework in a series of experiments in a simulation of a conference room currently under physical construction, showing how the controller can be easily modified to explore different lighting behaviors and energy use tradeoffs. The result of each experiment is a computer-generated animation of the lighting in a room over time from a single viewpoint, accompanied by estimated measurements of source input, light sensor output, and energy usage. A secondary objective is to match the simulation as closely as possible to a real physical environment with physical electric light sources and sensors. We demonstrate this calibration in a highly-controlled lighting research environment, showing how measurements of source and sensor specifications enable the output of the virtual sensors in the simulation to match the outputs of real sensors in the physical room when applying the same control law in both cases. Our research is aimed at both lighting designers seeking to quantitatively predict real-world controller behavior, and control algorithm researchers seeking to visualize results and explore design tradeoffs in realistic use cases. Furthermore, these simulation tools can aid in the benchmarking of candidate daylighting and lighting control algorithms for a given space.
\end{abstract}

Keywords: smart buildings, smart lighting, lighting control systems, environmental simulation, computer graphics, daylighting, energy harvesting

\footnotetext{
${ }^{*}$ Corresponding author

Email addresses: jial@rpi.edu (Li Jia), afshas@rpi.edu (Sina Afshari), mishrs2@rpi.edu (Sandipan Mishra), rjradke@ecse.rpi.edu (Richard J. Radke)
} 


\section{Introduction}

Architects routinely create photorealistic computer-generated still images of unbuilt environments at different seasons and times of day to pre-visualize design possibilities for clients. These renderings are generally meant to be evocative rather than physically accurate, and play little role in subsequent phases of construction. Similarly, lighting designers or manufacturers of lighting control systems may create computer-generated animations of the lights in a room dimming or turning off in response to changing sunlight or occupancy, for example as an argument for a potentially energy-saving controller. These renderings are generally not customized to a particular space and the lighting control may again be illustrative rather than carefully computed. In practice, specific choices about lighting design, such as the type, number, and position of fixtures and the lighting controller that governs the fixtures' behavior, are deferred to late in the design process, after enclosure systems, glazing, and shading devices have been decided. This prevents the early exploration of lighting technologies that could have a significant impact on energy use and human comfort.

The Energy Information Administration estimated that in 2011, $12 \%$ of the total electricity consumption in the United States was consumed by lighting, comprising about 186 billion $\mathrm{kWh}$ for residential lighting and about 275 billion $\mathrm{kWh}$ for commercial lighting [1]. In early literature, Lee and Selkowitz [2] emphasized that dynamic, responsive lighting controls that are customized to an environment can demonstrably reduce energy use and improve human comfort. Roisin et al. [3] reported a large range of energy savings (from 16\%-76\%) in their survey of various controlled lighting systems. Similarly, Singhvi et al. [4] reported $25 \%$ and $33 \%$ energy savings in cases without any comfort loss and with $7 \%$ comfort loss for the occupants, respectively.

Advanced lighting controllers are expected to have the fastest adoption and the most impact on energy savings in the commercial sector (e.g., office buildings and hospitals). In addition to reducing lighting energy consumption, incorporating appropriate daylighting and feedback control into building design enables comfortable and healthy architectural spaces $[5,6,7,8]$. For example, natural daylight affects human circadian rhythm, and exposure to windows has been shown to improve worker health and productivity $[9,10,11]$.

To reach their full effectiveness, advanced lighting controls should be incorporated early in the design phase, since they may affect choices such as fixture placement, glazing transparency, or window shading design. Pre-visualizing the realistic behavior of a lighting controller in a particular space in response to daylight and occupancy, as opposed to hypothetical behavior in a generic space, could mitigate complaints of clients whose employees are irritated by the controller behavior or turn off the controller entirely, destroying promised energy savings. Furthermore, with appropriate calibration to real-world physical spaces, a simulation tool could also be used as a benchmarking mechanism for evaluating the performance of lighting control systems in terms of the quality 
of light generated and energy savings achieved, without the need for replicating the actual physical space hardware. This paper presents a first step in the evaluation of such interactive pre-visualization.

The primary objective of this paper is to evaluate the capability of a combination of offline and online simulation to easily validate and tune the parameters of a candidate lighting control algorithm for a given space. The purpose is to qualitatively evaluate design choices and guide the selection and positioning of sources and sensors. We demonstrate this capability in a series of experiments in a digital simulation of a conference room currently under physical construction, showing how the controller can be easily modified to explore different lighting behaviors and energy use tradeoffs. The result of each experiment is a computergenerated animation of the lighting in a room over time from a single viewpoint, accompanied by estimated measurements of source input, light sensor output, and energy usage.

A secondary objective is to match the simulation as closely as possible to a real physical environment with physical electric light sources and sensors. We demonstrate this calibration in a highly-controlled lighting research environment called the Smart Space Testbed, showing how measurements of source and sensor specifications enable the output of the virtual sensors in the simulation to match the outputs of real sensors in the physical room when applying the same control law in both cases. The contributions are aimed at both lighting designers seeking to quantitatively predict real-world controller behavior, and control algorithm researchers seeking to visualize results and explore design tradeoffs in realistic use cases.

Section 2 generally overviews related research on simulation tools for lighting, advanced lighting control algorithms, and the ways in which such algorithms are validated. Section 3 describes the experiments undertaken in this paper and their objectives. Section 4 details the methodology developed to realize the experimental design, including a proposed simulation framework, an advanced lighting control algorithm, and a process for integrating the two. Section 5 reports the results of the experiments and discusses the iterative design process. Section 6 concludes the paper with challenges and directions for future work.

\section{Background and Related Work}

Section 2.1 presents a brief survey of simulation software tools used for lighting design and other related research. Specifically, we discuss the advantages and disadvantages of such tools with respect to the problems we study in the rest of the paper. Section 2.2 overviews approaches to the design of advanced lighting control algorithms. Finally, Section 2.3 discusses methods by which such algorithms are typically validated.

\subsection{Simulation Tools for Lighting Design}

Architects and lighting designers can choose from several different simulation programs to evaluate building envelope performance over various time 
scales and weather conditions $[12,13,14]$. Radiance, an open-source software package based on raytracing technology, is the most widely used lighting design tool, enabling lighting design, simulation, analysis, and pre-visualization [15]. A survey by Reinhart and Fitz [16] reported that among 42 daylight simulation tools used by participants, more than $50 \%$ were based on Radiance. The main disadvantages of using Radiance directly are its command-line interface and the effort required to describe the scenes of interest. Popular alternatives are the 3ds Max and Maya software packages by Autodesk, which enable the creation and visualization of detailed environmental models with complex texturing and lighting $[17,18,19]$. Rhinoceros (Rhino) is a similar 3D simulation tool that is easy to use and provides photorealistic rendering [20]. Most of the models created in these commercial programs are cross-compatible. To create an image, each simulation tool requires a renderer, which models or approximates the interaction between synthetic light sources and three-dimensional geometry; the most common renderers are Mental Ray, V-Ray, and Beast. Renderers chiefly differ in the degree to which they can achieve photorealism and the time it takes to obtain an image. Some simulation tools support multiple renderers.

In spite of the development of a wide array of lighting simulation tools, there are several key issues that impede their use in academic or professional practice for lighting simulations, including visualization capability, geometry and material complexity, spatial and temporal dimensions of daylight, and realtime performance feedback $[12,21]$. Several simulation tools require extremely long computation times, especially those that use raytracing technology. Also, there is little agreement on the definitions of building and performance assessment methods, so the simulation platforms are isolated, which makes validation, benchmarking and collaboration difficult [13]. It is desirable for dynamic lighting simulation tools to include time-varying, climate-based performance analysis capabilities that encourage an interactive, highly visual, and creativity-promoting design exploration process [22]. Reinhart and Wienold [23] presented a design analysis based on simulation that considers annual daylight, energy use, and human visual comfort, whose results could be shown in a "daylighting dashboard" for non-experts. MIT's Lightsolve supports interactive daylighting design that involves considerations of time and weather variations, though there is little variation in the tested daylighting systems [24, 25].

Our framework is designed to be interactive (in the sense that the user can easily modify choices about lighting controller design), which is exactly the purpose of platforms for designing computer games. The Unity3D game engine is well-suited to changing environments, importing external data, measuring and controlling objects' properties in real time, and human-machine interaction with scripting. In addition to its primary use for game design, Unity can be more broadly applied to research, design, and visualization of 3D environments. Wang et al. [26] proposed to use the Unity game engine as a virtual reality platform on a website for visitors to interactively view geographic information. Zyda [27] studied the trend of using visual simulation and games for interactive training and education. Indraparastha and Shinozaki [28] developed a virtual environment and investigated the use of the Unity game engine in an 
Table 1: Comparison of lighting simulation tools

\begin{tabular}{|l||l|l|l|l|l|}
\hline & Radiance & Maya & 3ds Max & Unity3D & Rhino \\
\hline \hline $\begin{array}{l}\text { Geometry/ } \\
\text { Material } \\
\text { Complexity }\end{array}$ & High & High & High & Low & High \\
\hline Area Light & High & High & Low & High & High \\
\hline Daylight & High & Low & High & Low & High \\
\hline Animation & None & High & Low & High & None \\
\hline $\begin{array}{l}\text { Real-time } \\
\text { Interaction }\end{array}$ & None & None & Low & High & None \\
\hline Scripting & High & High & Medium & High & Medium \\
\hline Ease of use & Low & Medium & Medium & High & High \\
\hline
\end{tabular}

urban design study. Experiments in simulated environments are often used to validate algorithms, such as those used for tracking and surveillance. Jia and Radke [29] proposed an environmental simulation method using the Unity game engine to collect time-of-flight data in a synthetic environment and validate occupancy tracking and pose estimation algorithms. Qureshi and Terzopoulos [30] combined computer graphics, simulated humans with complex behavior, and computer vision algorithms to investigate wide-area surveillance algorithms for camera networks.

Table 1 summarizes our perspective on the main simulation tools related to lighting design discussed here, evaluated with respect to several factors. These include the allowable level of geometry and material complexity; the ability to realistically simulate area or panel lights; the ability to realistically simulate the daylight for given geographical coordinates, times of day/year, and weather conditions; the ability to create an animation of a 3D rendered scene; the capacity for real-time user interaction with the renderer; the capacity to integrate the simulation program with user-written code (e.g., scripts in $\mathrm{C}++$ or $\mathrm{C \#}$ ), and the overall ease of use. We subjectively rated the simulation programs on a coarse scale of None, Low, Medium, and High.

Our experiments require the simulation of area lights, accurate daylight simulation based on site position/orientation and time of day/year, direct interaction between simulation and control, animation of the rendered scene, real-time interaction, and an easy-to-use interface. These considerations led us to investigate a combination of three simulation programs: Maya, 3ds Max Design, and the Unity Pro 3D game engine, as discussed further in Section 4.1.

\subsection{Advanced Lighting Control Algorithms}

The goal of energy-aware lighting design is to achieve sustainability, low energy consumption and human comfort in built environments [21]. To accomplish this, a typical feedback lighting control system uses illumination measurements of an environment to determine suitable light fixture commands that optimize 
a cost function balancing light quality, energy consumption, and occupant comfort. The design of control algorithms for lighting is an active research area. Galasiu and Veitch [6] reviewed and studied occupant preferences involving luminous environments and control systems. Mozer [31] prototyped a neural network system that controls residential utilities, such as air conditioning, lighting, and water heating in a house. Selkowitz et al. [32] investigated the internetbased control of lights, blinds and glazings, which allowed dynamic and responsive control of solar gain as well as daylight use. Singhvi et al. [4] proposed an intelligent building control strategy using mobile wireless sensor networks to optimize user comfort and energy consumption. Wen et al. [33] integrated wireless sensors and actuators to maximize the accuracy and robustness in intelligent daylighting systems for commercial buildings. Aldrich et al. [34, 35] used linear and nonlinear optimization techniques to increase the photometric characteristics of a color-tunable multi-channel LED light source while minimizing the energy consumption. Afshari et al. [36] proposed a feedback control design strategy for color-tunable LED lighting systems based on optimization of light quality, energy consumption and human comfort. It is critical to note that all these control algorithms aim to optimize a cost function that balances comfort and performance with energy cost.

\subsection{Validation Strategies for Lighting Control Systems}

The studies cited in Section 2.2 unanimously reiterate the importance of feedback control for energy-efficient lighting systems. However, such results are inevitably tied to a specific experimental testbed, which makes benchmarking difficult. For example, Mozer [31] used a three-room schoolhouse for implementation and validation of a feedback control algorithm for optimizing energy use and user discomfort. Sheng et al. [13] used a heliodon, a 1:0.25 scaled physical model of a space with a rotating light source for testing a daylighting illumination scheme. Wen et al. [33] tested their intelligent daylighting system in an office space with six desks and six dimmable light fixtures. Selkowitz et al. [32] implemented electrochromic windows for dynamic facades and daylighting in a three-room facility at the Lawrence Berkeley National Laboratory in Berkeley, California. Singhvi et al. [4] demonstrated their intelligent lighting control using sensor networks on a testbed consisting of sensor motes and standard table lamps in a small bench-top setup. Aldrich et al. [34] validated their intelligent control algorithm on an office desk with one sensor board and four commercial white-point adjustable luminaires.

The key challenge in establishing and comparing the performance of all these control algorithms is the lack of a uniform benchmarking tool that can be used by the control design community for validation and verification. In order to design the daylighting and lighting control algorithms, the physical space itself must be available for implementation and testing, which may be impractical in many cases. In [32], Selkowitz et al. reiterated the need for better simulation tools that can be tested and integrated for lighting control.

In addition to validation, the typical visualization and quantification of results in these studies is through time plots of energy measurements and measured 
light fields (such as lux, spectral intensity content) at specified discrete points in the lighting controlled space. It is clear that the perception of illumination by the occupant transcends a point-wise understanding and evaluation of the light field. In reality, visualization tools are critical to evaluate, analyze, and present the performance of any given lighting control algorithm.

We therefore require simulation tools for building lighting systems that enable flexible, rapid, and accurate design, analysis, and algorithm validation. With such a simulation tool, it will be possible to pre-visualize the performance of the control algorithm. Furthermore, these simulation tools can aid in benchmarking of various alternative daylighting and lighting control algorithms.

\section{Objectives and Experimental Design}

The primary objective of this paper is to demonstrate the capability of a combination of offline simulation and online simulation for interactively validating and tuning the parameters of a candidate lighting control algorithm for a given space. The purpose is to qualitatively evaluate design choices and guide the selection and positioning of sources and sensors. The secondary objective is to match the simulation as closely as possible to a real physical environment with physical electric light sources and sensors. Finally, we envision that through this simulation tool, candidate control algorithms can not only be efficiently designed but also benchmarked through comparisons of energy consumption, illumination quality, and other metrics.

We validate our contributions towards these objectives with four main experiments, summarized as follows:

1. Simulation of a large $\left(11^{\prime} \times 35^{\prime} \times 9^{\prime}\right)$ conference room (Figure 1a). This conference room is currently under construction and is being designed to enable highly advanced sensing, lighting, and control systems. The simulation accurately reflects the geometry of walls, windows, and furniture in the space under construction, and is roughly accurate in terms of the varying material properties (e.g., color, reflectivity and transparency) of surfaces in the environment.

The conference room is simulated with $602^{\prime} \times 2^{\prime}$ light panel fixtures distributed across the entire ceiling, and a downward-pointed color sensor at the center of each panel. The objective of this experiment is to show how design iterations using the simulation framework allow the parameters in a candidate control algorithm to be easily tuned to achieve a lighting designer's vision for fidelity to a given setpoint, color consistency, brightness consistency, and energy usage.

2. Simulation of the same room geometry as in Experiment 1, but replacing the ceiling lighting with 16 simulated Cree CR24 $2^{\prime} \times 2^{\prime}$ architectural LED troffers. This more closely resembles the lighting solution for the actual space. The objective of this experiment is to show that the tuned parameters from the lighting configuration in Experiment 1 can be carried over to a different lighting configuration and achieve similar performance. 
3. Simulation of the same room geometry and interior lights as in Experiment 1, but using a different, spatially-varying desired light field based on simulated occupants. The objective of this experiment is to simulate a use case with substantial potential energy savings and demonstrate that the candidate control algorithm is still effective.

4. Simulation of a small $\left(8^{\prime} \times 12^{\prime} \times 8^{\prime}\right)$ lighting prototyping environment called the Smart Space Testbed (Figure 1b). This is an existing experimental room, typically used to explore adaptive lighting strategies in a highly controlled environment. It includes a variety of repositionable color sensors, 12 wirelessly controlled multispectral LED lights each with a circular diffuser in a cone-shaped can, and a $2.3^{\prime} \times 4^{\prime}$ panel light in the middle of the ceiling that can simulate skylight. The room contains no windows to the outside or daylight.

The objective of this experiment is to demonstrate that with careful modeling of the actual light sources and color sensors based on measured data (e.g., cone angle, spectral sensitivity, color channel crosstalk), the simulation of a candidate control algorithm can be made to match physical reality both qualitatively and quantitatively, supporting the use of the simulation tool for making accurate predictions of lighting system behavior.

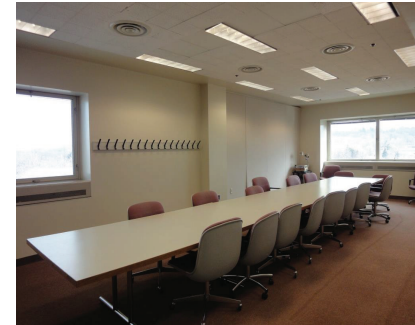

(a)

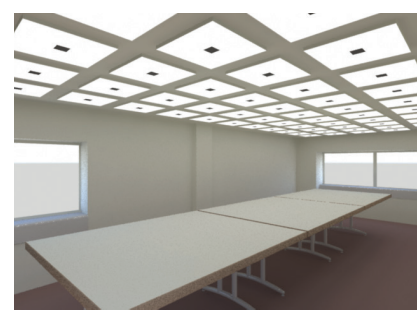

(c)

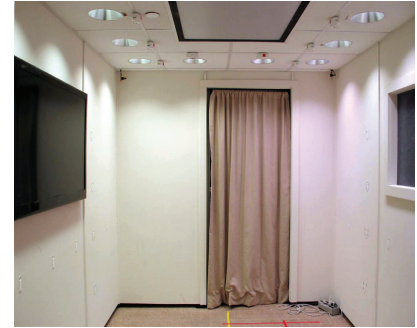

(b)

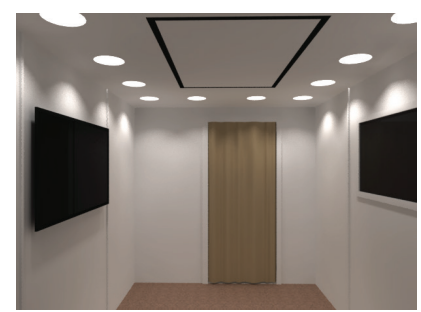

(d)

Figure 1: The experimental environments in real life (a-b) and in simulation (c-d). (a,c) The conference room. (b,d) The Smart Space Testbed.

We note that while we instantiate our simulations with particular choices of environments, sources, sensors, and control algorithms, the contributions of the paper are not specifically tied to these choices. We discuss the procedure and challenges of extending the approach to different choices in Section 6 . 


\section{Methodology}

In this section, we describe our proposed methodology for pre-visualizing lighting controller behavior in a specific space. Section 4.1 discusses our approach to simulating the space, using both an offline process to generate raytraced lightmaps and an online process to visualize the controller behavior. Section 4.2 summarizes the controller used in this study, previously proposed in [37]. Finally, Section 4.3 describes how the simulation and controller are integrated into a common framework.

\subsection{Simulation Tools}

As discussed in Section 2.1, the software tools Maya, 3ds Max Design, and Unity Pro all play a role in our simulation framework. We chose Maya for its ability to realistically simulate lighting panels with area lights that evenly emit photons from the panel using the mental ray plugin. 3ds Max Design has a superior daylight system that allows the specification of weather data, geographical location, date, and time. It also uses portal lights for global illumination, which allow the sun and sky light from windows to stream into the room. Unity Pro is well-suited to changing environments, importing external data, and measuring and controlling objects' properties in real time with scripting, making it an ideal lighting controller simulation platform.

First, we built detailed, accurately-scaled room models in Maya for each of the experimental environments, including both the geometry and varying material properties (e.g., color, reflectivity and transparency) of surfaces such as walls, tables, chairs, floors and ceilings. Next, we created highly accurate off-line renders for the lighting due to the panel lights (using Maya) and daylight (using $3 \mathrm{ds}$ Max), using their sophisticated algorithms for creating and visualizing global illumination. Figures $2 \mathrm{a}$ and $2 \mathrm{~b}-\mathrm{c}$ show respective examples, using the correct geographical location and orientation of the conference room (and default sunny weather).
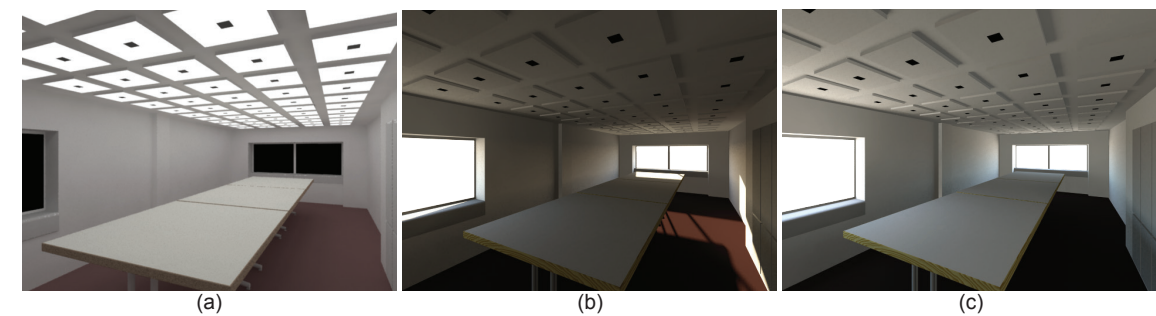

Figure 2: (a) Rendering in Maya of lighting due to ceiling fixtures only. (b,c) Rendering in 3ds Max due to daylight in Troy, NY on 1 June 2013 at (b) 7:00am and (c) 2:00pm.

It can take a substantial amount of time to render a near-photorealistic image using global illumination for a single frame. For example, creating the image in Maya illustrated in Figure 2a took about 2 hours, while creating the 
images in 3ds Max Design illustrated in Figure 2b-c each took about 2min, all on a modest desktop computer (3.6 GHz, 8 GB RAM). However, since our interest is in exploring different control algorithms for the same external lighting conditions, we save the rendered lighting in texture files and reuse them. These textures are called "lightmaps", each of which is a $1024 \times 1024$ image containing all the geometric faces in the environment and indicating the brightness on each surface. The lightmap is independent of a particular camera perspective. Figure 3 a shows the lightmap resulting from the daylight render in Figure 2c. We can then import each stored lightmap into a room model in Unity, as illustrated in Figure $3 \mathrm{~b}$.
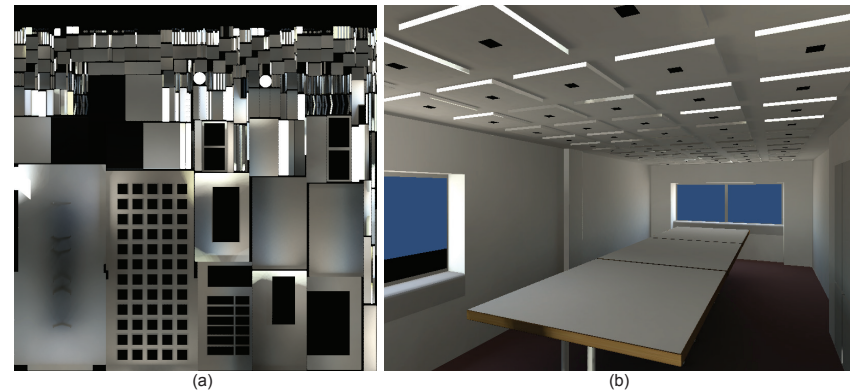

Figure 3: (a) Lightmap corresponding to Figure 2c (daylight in Troy, NY on 1 June 2013 at 2:00pm). (b) Conference room simulation using the imported lightmap in Unity.

Figure 4 summarizes our overall procedure for pre-computing the necessary materials to simulate a room with given environment model and light configuration. While this procedure may be time-consuming, it only happens once, prior to interactive user exploration of lighting controller behavior.

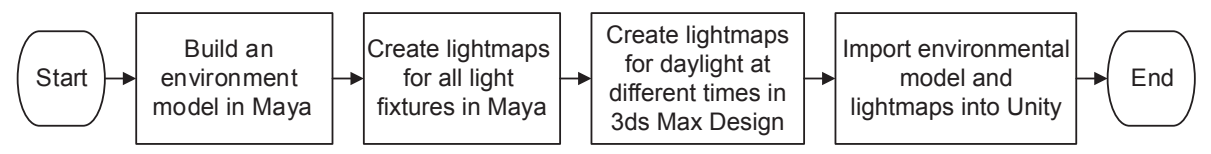

Figure 4: Pre-computation procedure for lighting simulation.

We must also place and model the color sensors in the environments used for feedback in the lighting controllers. For the conference room testbed under construction (Experiments 1-3), we simulate color sensor readings by placing a single downward-looking orthographic camera on the simulated ceiling, and calculate virtual color sensor responses by averaging RGB values in circles with specified radii as illustrated in Figure 5a. The color sensor readings are used as inputs to the lighting control algorithm, just as if they came from physical sensor readings. Thus, the feedback control loop is virtually simulated.

In the Smart Space Testbed, our research in advanced lighting controllers 


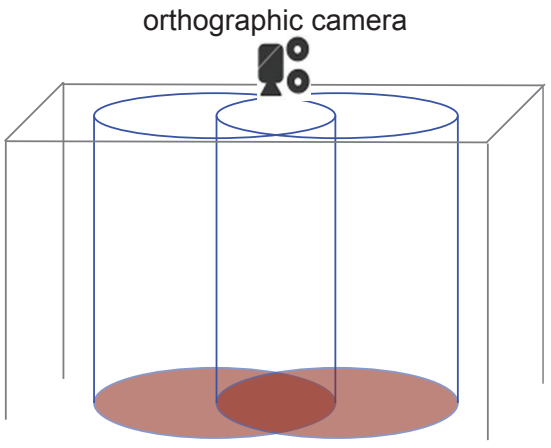

(a)

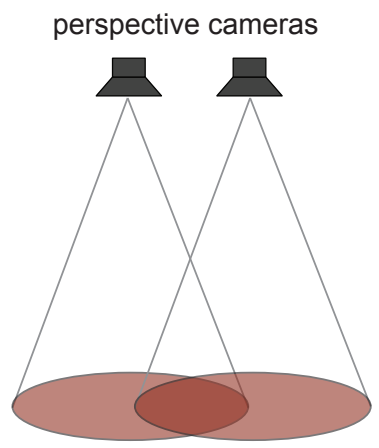

(b)

Figure 5: (a) In Experiments 1-3, color sensors are simulated by averaging colors in an image from a single orthographic camera. (b) In Experiment 4, we more accurately simulate color sensors using images from several perspective cameras.

uses repositionable Seachanger wireless "Color Bug" sensors to measure local RGB intensity and illuminance. Our goal in Experiment 4 is to approximate the true responses from these sensors as closely as possible in the simulation, which we refer to as calibration. To build an accurate model, we measured the angular sensitivity of a Color Bug using a spectroradiometer from $-90^{\circ}$ to $90^{\circ}$ in $10^{\circ}$ increments, as illustrated in Figure 6a. The sensors in the simulation for Experiment 4 are modeled using downward-pointed perspective cameras, slightly offset from the light sources, whose resulting images are filtered with the measured angular sensitivity profile to produce a single RGB reading per sensor, as illustrated in Figure 5b.

We also noticed that the RGB colorspaces of the Color Bug and the LED lights in the room were different, requiring a transformation in the simulation to map the lightmap values into the correct sensor readings. We modeled this as a linear transformation, which we fitted with least-squares optimization using the measured data from the real sources and sensors. The resulting $3 \times 3$ matrix, which multiplies RGB values from the lightmaps to produce RGB values of the simulated sensors, is given in Figure 6b.

\subsection{Lighting Control Design}

In this section, we present an application of the simulation tool for enabling design iterations for a feedback control algorithm. The optimal lighting feedback control design problem is defined as determining the intensities of light sources to minimize an objective function, such as the one developed in [37]. The determination of the optimum of this cost function in the presence of modeling uncertainty, evolving ambient light disturbances, and feedback measurement noise is the crux of the lighting control algorithm.

It is key to note that while the design process here is directed towards the control law developed in [37], the primary contribution of this manuscript is 


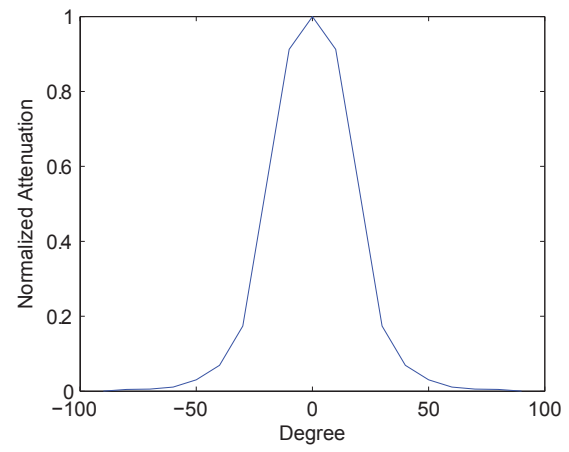

(a) $\left[\begin{array}{lll}0.225 & 0.008 & 0.001 \\ 0.010 & 0.091 & 0.006 \\ 0.005 & 0.035 & 0.029\end{array}\right]$

(b)

Figure 6: (a) Normalized measured angular sensitivity for Color Bug sensor. (b) Measured colorspace transformation matrix for Color Bug sensor.

the use of the dynamic lighting simulation tool for designing parameters of the optimization function. Thus, a similar procedure may be followed for designing and tuning parameters of the control laws developed in the literature [4,34,38].

The system description used for this lighting control algorithm is based on the light transport model [39], which relates the light intensities of a set of $N$ sources (of specified spectra) to the RGB color readings at $M$ locations of interest. This model is static (i.e., no temporal dynamics) and linear [39]. Succinctly, this model relates the input source intensities $u\left(\in \mathbb{R}^{N}\right)$ and ambient (disturbance) light $d\left(\in \mathbb{R}^{M}\right)$ to measured light intensities $y\left(\in \mathbb{R}^{M}\right)$ as:

$$
y_{t}=G u_{t}+d_{t}
$$

where the subscript $t$ has been added to emphasize that this relationship holds for the time instant $t$.

The cost function $(J)$ consists of the weighted sum of a light quality metric and a uniformity metric for occupant comfort, and an energy consumption metric. The light quality metric penalizes the difference between the desired light field and the generated light field, while the uniformity metric enforces a penalty on the inhomogeneity of color output from adjacent light fixtures. Finally, the energy metric represents the total energy consumption of the light fixtures. Thus, the optimal lighting source intensities $(u)$ can be obtained by solving the following optimization problem:

$$
\begin{array}{ll}
\underset{u}{\operatorname{minimize}} & J=\mu_{Q}\left(y_{d e s}, y\right)+\alpha_{U c} \cdot \mu_{U c}(u)+\alpha_{U b} \cdot \mu_{U b}(u)+\alpha_{E} \cdot \mu_{E}(u) \\
\text { subject to } & \mathcal{F}(y) \in \mathcal{S} \quad \text { and } y=G u+d
\end{array}
$$

where $y_{\text {des }}$ is the desired light field, $y$ is the measured light field, $\mu_{Q}(\cdot, \cdot)$ measures the quality of the match between two light fields, $\mu_{U}(\cdot)$ measures the 
non-uniformity between light outputs from fixtures, and $\mu_{E}(\cdot)$ measures the total energy consumed by the fixtures. We model the energy term as a linear function of the light fixture input vector [34], the coefficients of which account for the efficiencies of different color channels. It is key to note that this energy consumption model is dependent on the specific type of LED driver used.

$\alpha_{U c, U b}$ are adjustable weighting coefficients determining the relative cost of non-uniformity of LED color and brightness, respectively, versus light quality. Similarly, $\alpha_{E}$ is the relative penalty on energy consumption. Finally, $\mathcal{F}(\cdot)$ is a function that characterizes the comfort of a generated light field for the human eye and $\mathcal{S}$ is the set of all comfortable (acceptable) lighting conditions. Since $d$ is unknown and time-varying, feedback methods are needed to solve (2) online. The typical feedback control law is obtained by computing the gradient of the cost function in (2) from sensor measurements $\left(y_{t}\right)$, and is of the form

$$
u_{t+1}=F\left(u_{t}, y_{t}, y_{d e s}\right)
$$

Such optimization-based feedback control techniques can deliver significant energy savings while optimizing light quality and occupant comfort. However, a key challenge in the design of the control algorithm lies in the construction of the cost function itself. For example, it is unclear how the choice of the weights $\alpha_{U c, U b}$ and $\alpha_{E}$ affect the overall aesthetics of the room or the color quality degradation. Furthermore, although the quality metric $\mu_{Q}$ is meant to evaluate the light field as a whole, in implementation this light field is inferred only through discrete intensity measurements from color sensors at specific locations. As a result, the control system's performance is heavily tied to the locations of these sensors and blind to the actual light fields generated. For both these reasons, it is critical to develop simulation tools that enable

- assessment of a designed cost function and thus iterative choice of the relative weights of various terms in the cost function, and

- evaluation of sensor measurements and desired setpoints and their correlation to generated light fields.

\subsection{Integrating Simulation and Control}

We now describe how we integrate the lighting simulation described in Section 4.1 with the control algorithm described in Section 4.2, which requires several steps:

1. Modeling: Create the geometry, material properties, and lightmaps for the desired environment as described in Section 4.1. The result is a Unity simulation that can render a photorealistically lit space corresponding to any position of the sun and any single light fixture.

2. Initialization: Determine the (possibly time-varying) desired light field for the environment, which may depend on the occupants' positions and needs. We also specify initial RGB intensity values for all the light fixtures in the room. 
3. Light rendering: Combine the precomputed lightmaps to render the room lighting corresponding to the sun position and specified fixture intensities.

4. Color sensor reading: Calculate synthetic color sensor readings by averaging RGB values from downward-looking cameras in the lit environment.

5. Lighting control: Input the color sensor readings to the control algorithm along with the desired light field to update the RGB intensity values for all the light fixtures.

6. Looping: return to Step 3.

We elaborate on Step 3. In Section 4.1 we discussed how the lightmaps due to each individual fixture and sun position are precomputed and stored. Since light transport is linear, we use superposition to create a composite light field out of these pre-rendered lightmaps to create an accurate lightmap for any combination of sun position and RGB values for each fixture.

In particular, suppose there are $M$ color sensors, $N$ light fixtures and $T$ different sun positions. For each of the $N$ fixtures, we set the RGB color to $[1,0,0]$, $[0,1,0]$, and $[0,0,1]$ (i.e., full red, green, and blue), render global illumination results for each channel, and collect the $3 M \times 1$ vector of color sensor readings for this scene (with no daylight). The color sensor readings due to each fixture are collected into a $3 M \times 3 N$ matrix denoted $C$. Similarly, for each of the $T$ sun positions, we compute the $3 M \times 1$ vector, denoted $d_{t}$, corresponding to the color sensor readings at time $t$ for this scene (with no fixture lighting).

Thus, when the RGB values of each light determined by the control algorithm at time $t$ are specified by a $3 N \times 1$ vector $u_{t}$, we calculate the corresponding $3 M \times 1$ color sensor reading $y_{t}$ as:

$$
y_{t}=C \cdot u_{t}+d_{t}
$$

We note this process is immediate, and generates accurate color sensor readings for any sun/fixture configuration even though the corresponding scene is never actually rendered. Thus, even though it may take several minutes or hours to render all the basis colormap images and collect color sensor readings (i.e., $C$ and $\left\{d_{t}\right\}$ ), the input-output relationship in the control loop is simply a low-dimensional matrix-vector product, allowing rapid exploration of different control strategies for a given space.

Since we ultimately want to visualize the changing illumination in the room due to the control algorithm, we also record the lightmap $l_{t}$ corresponding to the determined illumination at time $t$ (i.e., a $1024 \times 1024$ matrix), which like (4) is a linear combination of precomputed lightmaps using the control inputs $u_{t}$ as weightings. These are computed in a batch after the control simulation is complete, imported all together into Unity, and allow the resulting video to be accurately rendered in real time. The overall procedure for lighting control simulation and rendering is summarized in Figure 7.

For all the experimental results reported in this paper, we ran 30 iterations of the control loop between each simulation time step to allow the control algorithm to converge before switching to the next sun lightmap in the sequence. 


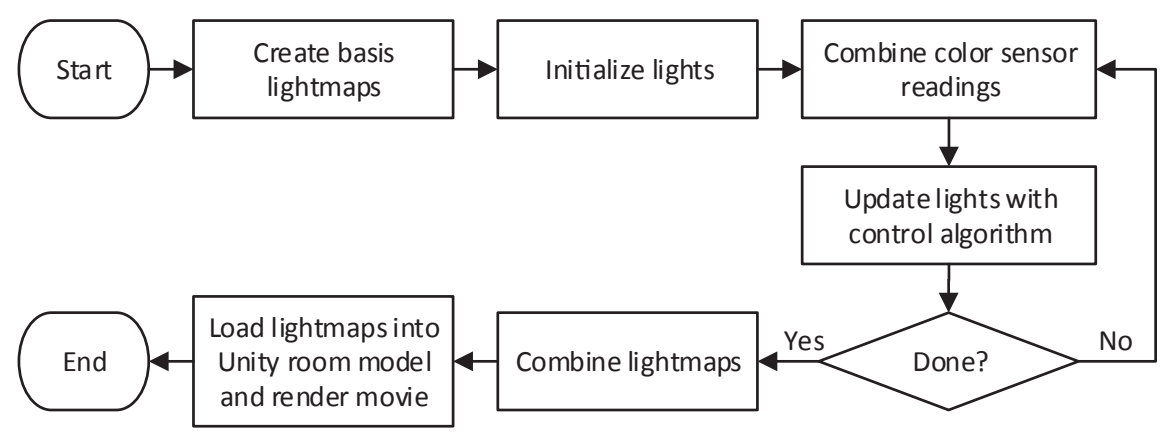

Figure 7: Procedure of real-time simulation with lighting control.

Convergence of the control algorithm from initial color sensor readings of $[0,0,0]$ only takes a few seconds of real time. Therefore, it is reasonable to assume time-scale separation of the action of the control algorithm and the change in the ambient sunlight pattern.

\section{Experimental Results}

In this section, we discuss the results of visualizing and tuning the lighting controller behavior through a sequence of design iterations in the four experiments introduced in Section 3.

\subsection{Experiment 1: Conference Room Simulation}

We first discuss the controller tuning process in the basic conference room simulation. Figure 8a shows an example of this environment with lighting from all LED fixtures as well as daylight. Figure $8 \mathrm{~b}$ shows a diagram of the room from above, indicating the layout and numbering scheme of each LED/color sensor pair. The left and right sides of the diagram correspond to the near and far sides of the room in Figure 8a, respectively.

As described in Section 4.3, we rendered 60 lightmaps for the LED fixtures and 144 lightmaps (each separated by 10 minutes) for sun positions, corresponding to a 24-hour day on 1 June 2013 in Troy, New York, USA. Each channel of the LED input is measured on a scale from 0 (fully off) to 1 (fully on). We chose the RGB intensity for each LED fixture as $[0.48,0.5,0.52]$ and calculated the corresponding readings for each color sensor (which vary depending on their position in the room), thereby constructing the desired illumination setpoint vector $y_{d e s}$ in (2). Note that while the entries of $C$ in (4) are all in the range $[0,1]$, the color sensor setpoints and readings can be greater than 1 since the sources and sensors in this case are closely spaced.

With reference to the cost function (2), we conducted six controller design iterations with varying weights on chromaticity and brightness uniformity 


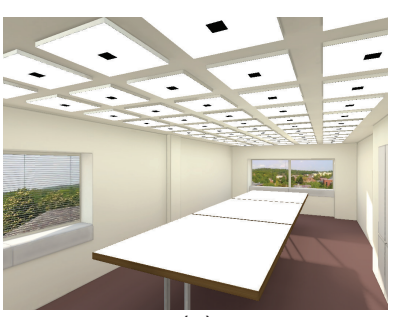

(a)

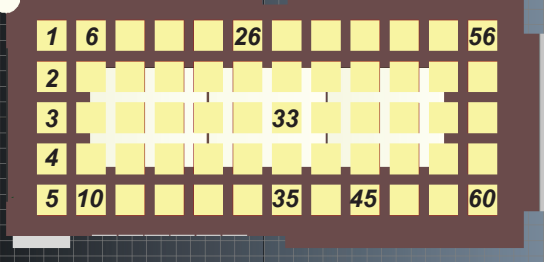

(b)

Figure 8: The configuration of Experiment 1. (a) Conference room simulation with lighting from LED panels and daylight. (b) Diagram of the room from above indicating light/panel layout.

$\left(\alpha_{U c, U b}\right)$, and energy usage $\left(\alpha_{E}\right)$. In practice, the iterations were separated by several days as the results were discussed and the objective function modified to produce controller behavior deemed to be desirable. The iterations are as follows, described in further detail below:

- Exp-1a: No disturbance, no weighting on energy $\left(\alpha_{E}=0\right)$

- Exp-1b: No disturbance, energy weighting $\alpha_{E}=0.1$

- Exp-1c: With disturbance, $\alpha_{E}=0$

- Exp-1d: With disturbance, $\alpha_{E}=0$, weight 1 on chromaticity uniformity $\left(\alpha_{U c}\right)$.

- Exp-1e: With disturbance, $\alpha_{E}=0, \alpha_{U c}=1$ and varying weights 0.001 , $0.01,0.1$ and 1 on intensity uniformity $\left(\alpha_{U b}\right)$.

- Exp-1f: With disturbance, $\alpha_{E}=0.1, \alpha_{U c}=1$ and $\alpha_{U b}=0.001$.

We first considered an empty room with no external disturbance from sunlight. The initial condition was a darkened room with all light fixture intensities set to $[0.1,0.1,0.1]$. In Exp-1a, we observed the lights quickly converging to the setpoint. When a weight on energy usage is added (Exp-1b), the illumination in the room at convergence is somewhat darker and the fidelity of the desired setpoint is compromised, as would be expected. After convergence, the inputs to LED-35 in Exp-1a and Exp-1b are $[0.48,0.5,0.52]$ and $[0.35,0.4,0.42]$, respectively. On the other hand, the energy usage in Exp-1b was $78 \%$ of that in Exp-1a. These results mirror what occurs when the control algorithm is implemented in a physical environment (see Section 5.4 and [37]).

Next, we ran the same control algorithm as Exp-1a, but added daylight as the sun moved throughout the sky (Exp-1c). We immediately observed that the controller failed to converge and produced non-uniform colorful patterns on the LED panels whose time-varying behavior was very visually distracting, as illustrated in Figure 9a. This led us to modify the cost function to encourage 
uniformity in chromaticity by setting $\alpha_{U c}$ to 1 in (2) (Exp-1d). The results are illustrated in Figure 9b. The panels are no longer randomly colored (that is, the uniformity term forces the R, G, and B channels to be almost identical for every fixture). However, the intensities of neighboring lights still differ sharply and can change quickly, which is still very visually distracting.
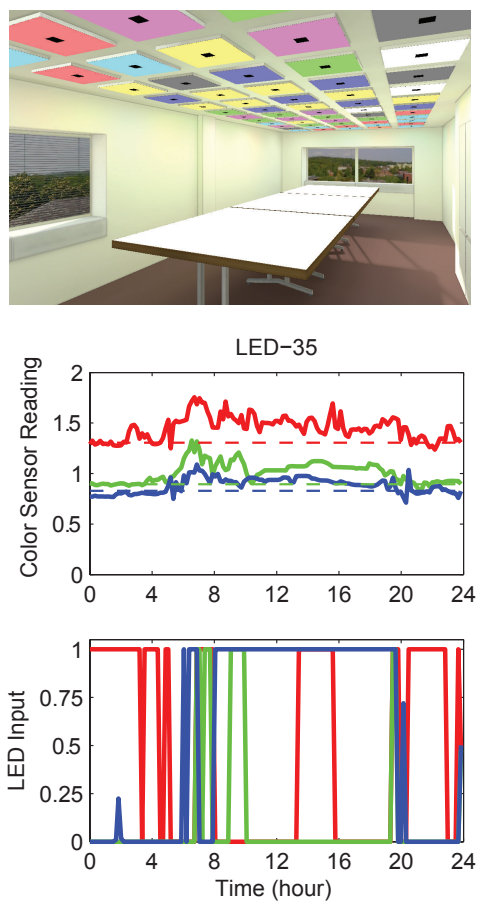

(a)

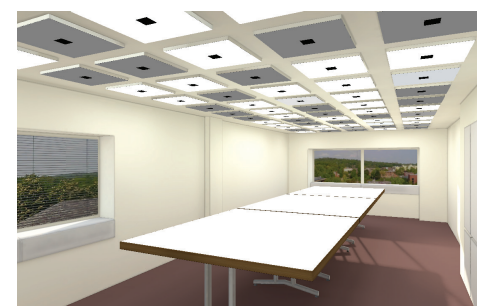

LED-35
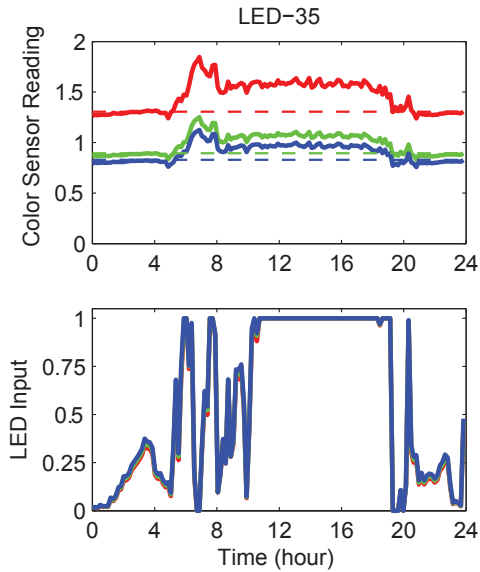

(b)

Figure 9: Control results for (a) Exp-1c and (b) Exp-1d, Top: visualizations of the room at 7:00am. Middle: color reading from sensor 35. Bottom: color LED input.

To address this issue, we modified the cost function to additionally encourage uniformity in intensity by making $\alpha_{U b}$ nonzero in (2) (Exp-1e). Figure 10 illustrates the results as $\alpha_{U b}$ is varied across $\{0.001,0.01,0.1,1\}$, keeping all other experimental parameters the same as in Exp-1d. The color input and sensor readings are graphed for two adjacent light fixtures, allowing the assessment of the effect of uniformity. We can see that as the parameter $\alpha_{U b}$ is increased, the "checkerboard" effect diminishes, until the entire ceiling effectively acts as a single dimmable fixture. We can see that despite the qualitative differences in each case, the control algorithm is able to drive the color sensor readings to the desired setpoint. We note that fixture 35 is directly above a bright patch of sun on the floor at 7:00am; the controller adapts to this disturbance and dims the fixture. In this case, an intensity uniformity weight of 0.001 seems reasonable for a lighting control system, which allows lights directly above a directly 
sunlit area to dim significantly (thus saving energy), while nearby lights above an indirectly lit area can remain bright.
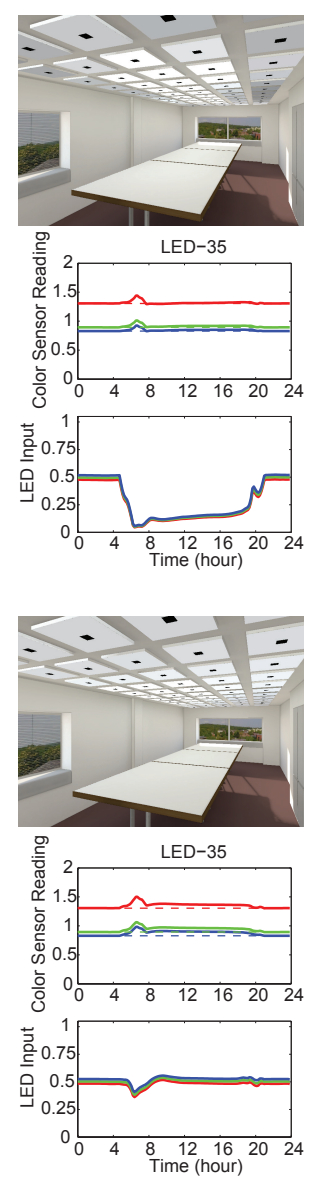

(a)
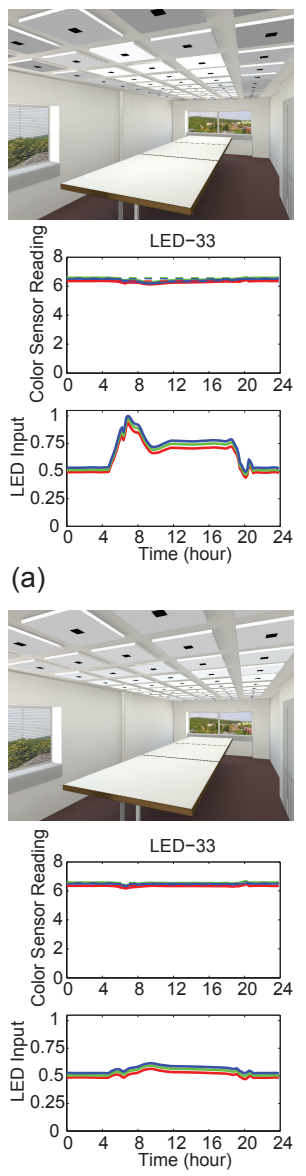

(c)

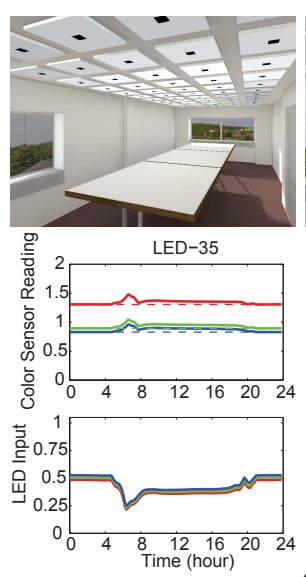

(b)
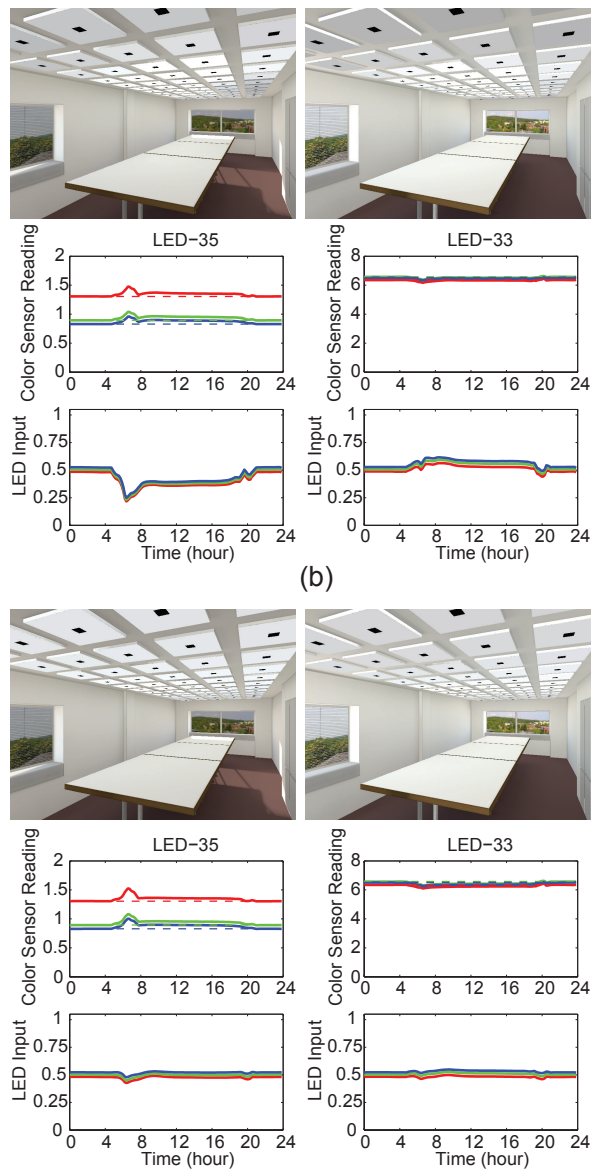

(d)

Figure 10: Control results for Exp-1e, with weights on intensity uniformity of (a) 0.001 (b) 0.01 , (c) 0.1 and (d) 1. In each subfigure, the top images are visualizations of the room at 7:00 and $2: 00 \mathrm{pm}$ and the bottom graphs plot color sensor readings and LED inputs for fixtures 35 and 33 .

We also measured the energy consumption for each of the different weights on intensity uniformity, as reported in Table 2. This consumption is measured as a percentage of the energy consumption in Exp-1a, averaged over the working hours of 8:00am to 8:00pm. That is, the Exp-1a controller does not dim the lights after convergence, and the lights are tuned to the desired setpoint $[0.45$, $0.5,0.52]$ all the time. Compared to this, the first Exp-1e lighting system would only consume $79 \%$ of the energy if some of the lights are automatically dimmed to take advantage of the sunlight. An even more advantageous comparison is to 
Table 2: Average energy consumption for different intensity uniformity weights in Exp-1e. Energy usage is normalized with respect to the usage of Exp-1a.

\begin{tabular}{c|cccc}
$\alpha_{U b}$ & 0.001 & 0.01 & 0.1 & 1 \\
\hline$\%$ energy & 79 & 83 & 84 & 85
\end{tabular}

a non-dimmable system in which all lights are either fully on (i.e., $[1,1,1]$ ) or fully off (i.e., $[0,0,0]$ ). The first Exp-1e lighting system would only consume $39 \%$ of the energy of a system that is fully-on during working hours.

We finally added the same weight as in Exp-1b to the energy usage term in the cost function, keeping the weight on chromaticity uniformity at 1 and the weight on intensity uniformity at 0.001 (Exp-1f). As in Exp-1b, the fidelity to the setpoint is compromised, but the energy usage during working hours relative to Exp-1a is reduced to $63 \%$ ( $31 \%$ compared to a fully-on system).

Please refer to the video accompanying this manuscript for a better appreciation of the controller design iterations described in this section.

\subsection{Experiment 2: Modified Conference Room Simulation}

In Experiment 2, we modified the conference room model to replace the dense panel lights with two rows of 8 troffers each, as illustrated in Figure 11a. This modification more closely resembles the lights that will be mounted in the physical space; our goal is to evaluate whether the controller design can easily be transferred to new configurations. Figure 11b shows a diagram of the room from above, indicating the layout and numbering scheme of each LED/color sensor pair. The left and right sides of the diagram correspond to the near and far sides of the room in Figure 11a, respectively.

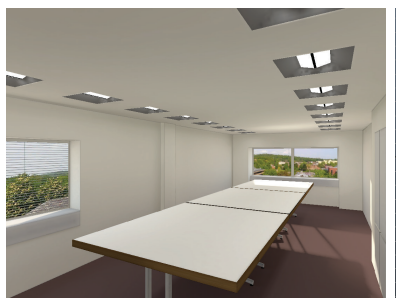

(a)

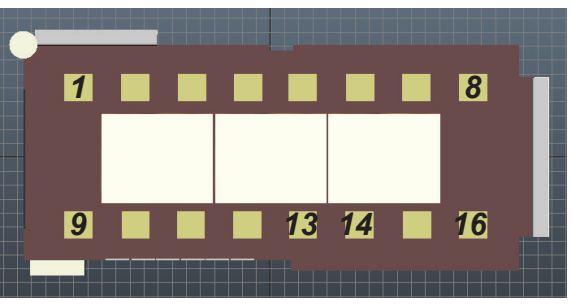

(b)

Figure 11: The configuration of Experiment 2. (a) Modified conference room simulation with lighting from LED panels and daylight. (b) Diagram of the room from above indicating light/panel layout.

As in the previous section, we rendered lightmaps for each of the 16 LED fixtures and 144 sun positions. As before, the setpoint for each fixture was $[0.48,0.5,0.52]$ and the setpoints for color sensor readings were calculated at initialization. We conducted two design iterations to test the lighting control algorithms in the new configuration: 
- Exp-2a: With disturbance, no weighting on energy $\left(\alpha_{E}=0\right)$, weight on chromaticity uniformity $\alpha_{U c}=1$, weight on intensity uniformity $\alpha_{U b}=$ 0.001 .

- Exp-2b: With disturbance, $\alpha_{E}=0, \alpha_{U c}=1$ and $\alpha_{U b}=0.05$.

Exp-2a is directly comparable to Exp-1e from Experiment 1; the experimental results are illustrated in Figure 12 for two times of day and two adjacent fixtures. As before, the controller succeeded with dimming a fixture (LED-14) above a bright patch of sunlight, but an adjacent fixture (LED-13) was brightened to achieve the desired color sensor readings, leading to a perceptually distracting non-uniformity between nearby lights.
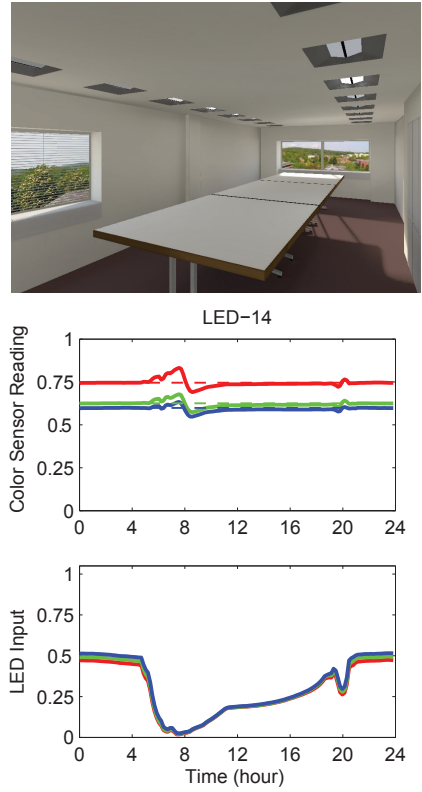

(a)

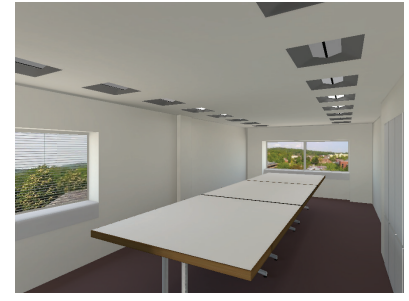

LED-13
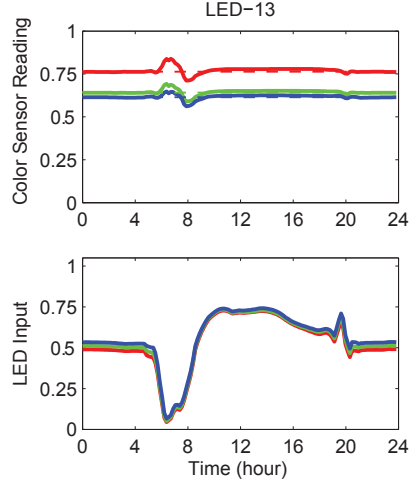

(b)

Figure 12: Control results for Exp-2a. Top: visualizations of the room at (left) 7:00 and (right) 2:00pm. Bottom: color sensor readings and LED inputs for adjacent fixtures (left) 14 and (right) 13.

We therefore increased the weighting on intensity uniformity to 0.05 (Exp2b); the experimental results are illustrated in Figure 13. Compared with Figure 12 , we can see the lights are more uniform as desired.

The energy consumptions (averaged as before over daylight hours and normalized with respect to the converged LED inputs without disturbance) for the two experiments were $50 \%$ and $55 \%$ for Exp-2a and Exp-2b, respectively. 


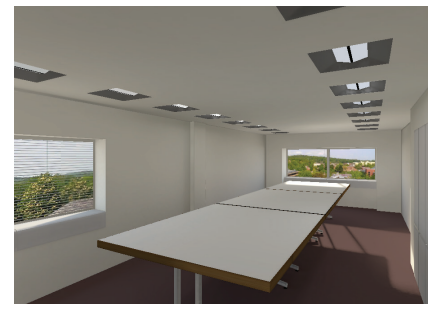

LED-14
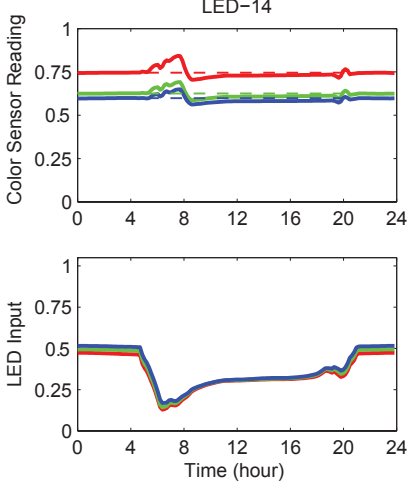

(a)

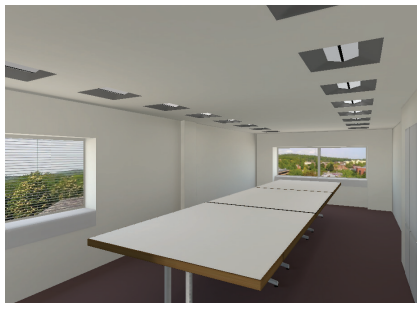

LED-13
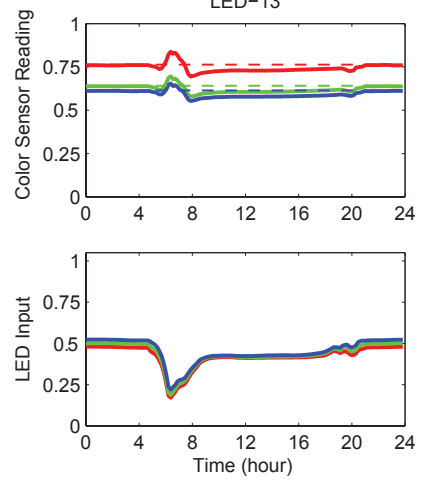

(b)

Figure 13: Control results for Exp-2b. Top: visualizations of the room at (left) 7:00 and (right) 2:00pm. Bottom: color sensor readings and LED inputs for adjacent fixtures (left) 14 and (right) 13. 


\subsection{Experiment 3: Occupancy-Specific Lighting}

Ultimately, we are interested in how advanced lighting controllers can be designed to intelligently react to the presence of occupants based on tracking or estimating their position. For example, lights could dim in regions of the room where no people are working, out of the line of sight of the occupants. In our previous work [29] we analyzed how downward-pointed, ceiling-mounted time-of-flight sensors could be used to monitor the position and pose of room occupants in real time. Our simulation easily allows us to simulate such sensors when human characters are introduced into the environments.

Figure 14 shows the configuration for Experiment 3, the 60-fixture conference room with pools of light centered around the seated occupants. The rest of the lights are mostly dimmed to provide low-level ambient lighting. In this case, the 5 bright lights (fixtures 22, 27, 28, 29 and 34) have LED input [1, $1,1]$ while the remaining lights have setpoint [0.04, 0.04, 0.04]. We used this lighting configuration to define the desired input and color sensor setpoints for the control algorithm. Sunlight disturbance was included in the experiment, and the controller parameters were specified as no weight on energy $\left(\alpha_{E}=0\right)$, weight on chromaticity uniformity $\alpha_{U c}=1$, and weight on intensity uniformity $\alpha_{U b}=0.001$.

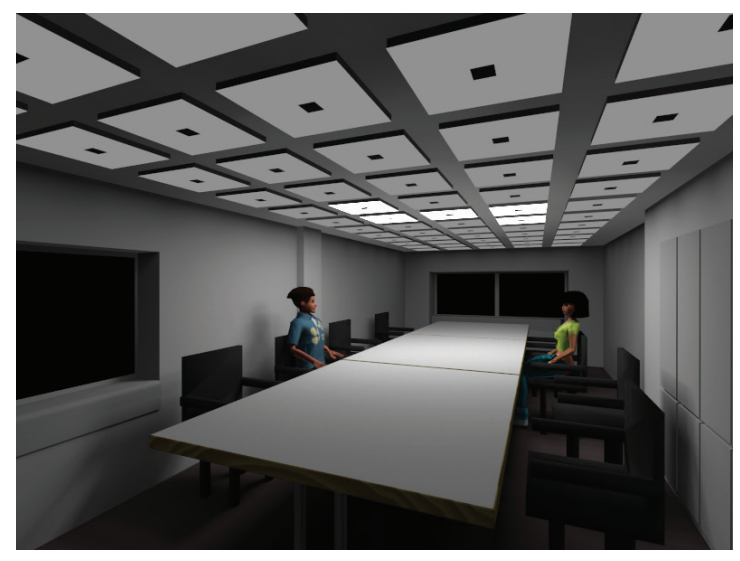

Figure 14: The configuration for Experiment 3, an example of non-uniform, occupancydependent lighting.

Figure 15 visualizes the illuminance resulting from the control algorithm at time instants 7:00am, 2:00pm and 10:00pm for Experiment 3. As expected, the lights above the seated people are almost fully on, and lights where people are not present are almost fully off.

Figure 16 shows the color sensor readings and LED inputs for several light fixtures. In (a) and (b), we averaged the values from the 2 fixtures above the seated male and female occupants, respectively, while (c) reports the values for a fixture far from the occupants. These results give a more quantitative view of 


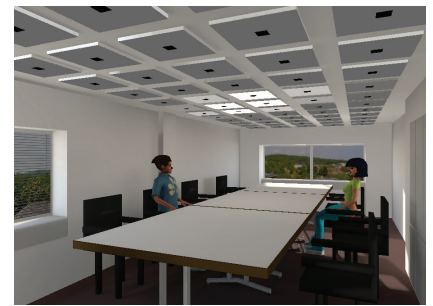

(a)

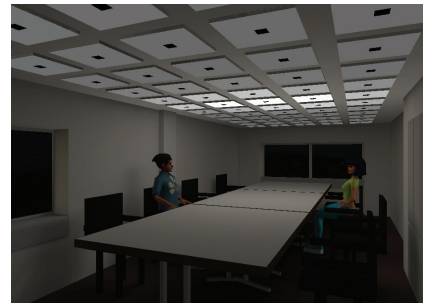

(c)

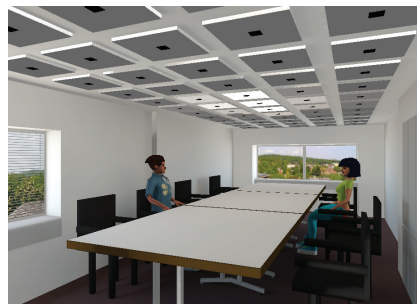

(b)

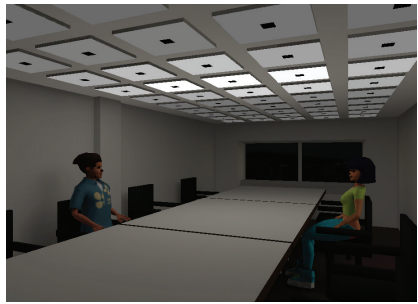

(d)

Figure 15: Visualizations of the control results for Experiment 3 at (a) 7:00am (direct sunlight beneath several fixtures), (b) 2:00pm (indirect illumination from sky light), and (c) 10:00pm (no daylight disturbance). (d) is a closer view of (c).

the control algorithm's behavior; for example, when direct sunlight contributes to the room illuminance (e.g., early morning above the female character), the fixtures are significantly dimmed (visible in Figure 15a).
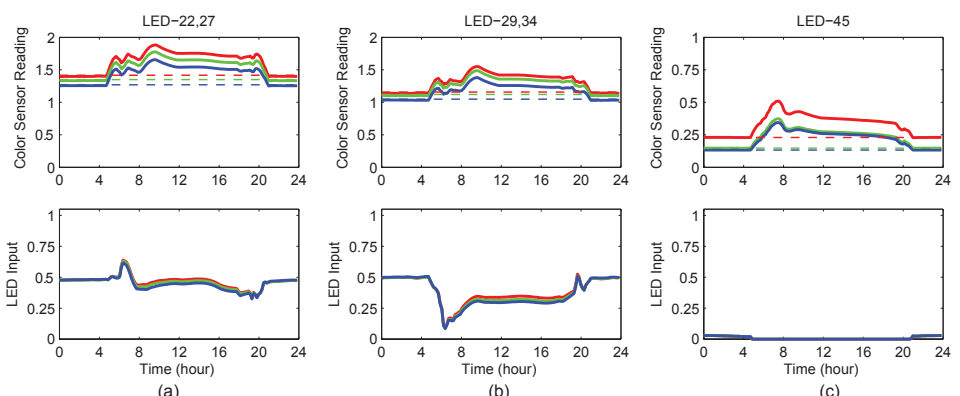

Figure 16: Color sensor readings and LED inputs for Experiment 3. (a) averages for fixtures 22 and 27 (above seated male), (b) averages for fixtures 29 and 34 (above seated female), (c) fixture 45 (far from seated people). Refer to Figure $8 \mathrm{~b}$ for fixture numbering.

We again computed the energy consumption during working hours. The energy consumption was $82 \%$ of the energy corresponding to the constant LED input illustrated in Figure 14, 19\% of the energy corresponding to the constant LED input illustrated in Figure 8a, and $9 \%$ of the energy corresponding to having the lights fully-on during working hours. These results suggest that 
substantial energy savings can be realized by customizing the lighting to the occupants, as is well-known [40, 4, 41].

\subsection{Experiment 4: Comparing the Real and Simulated Smart Space Testbeds}

Finally, Experiment 4 addresses the secondary objective of accurately matching the simulation LED source and color sensor readings to a physical space. The process of calibrating the response of the actual sensors in the Smart Space Testbed to the actual sources was described in Section 4.1.

Figure 17a shows the simulated Smart Space Testbed, and Figure 17b shows a diagram of the Smart Space Testbed from above, indicating the layout and numbering scheme of each LED/color sensor pair. The left and right sides of the diagram correspond to the near and far sides of the room in Figure 17a, respectively.

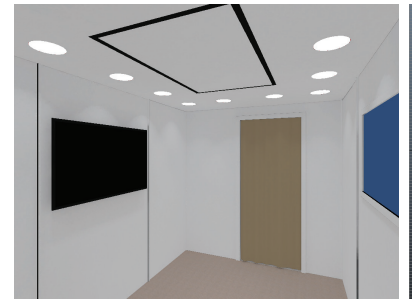

(a)

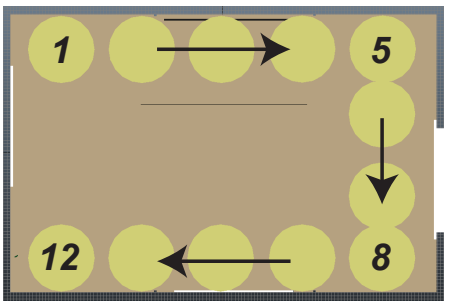

(b)

Figure 17: The configuration of Experiment 4. (a) Smart Space Testbed simulation with lighting from LED fixtures. (b) Diagram of the room from above indicating fixture layout.

We conducted a 60 -second experiment (144 frames of simulation). The initial condition is a dark room and the LED input setpoint is $[0.3,0.3,0.4]$ for all fixtures. External disturbance was not included in the experiment, and the controller parameters were specified with different weights on chromaticity uniformity and intensity uniformity $\left(\alpha_{U c}=1, \alpha_{U b}=0.01\right)$ and a weight on energy $\alpha_{E}$ that is 0 from $t=0$ to 30 seconds and $\alpha_{E}=0.04$ from $t=31$ to 60 seconds.

The top row of Figure 18 compares the color sensor readings and LED inputs for three situations: the actual measurements from the physical space (left column), the simulated measurements in Experiment 4 with the calibrated color sensor model (middle column) and the results of Experiment 4 using the noncalibrated color sensor model based on the orthographic camera used throughout Experiments 1-3 (right column). The bottom row of Figure 18 does the same for the normalized energy consumption.

We can see that the left and middle columns of Figure 18 are almost identical. On the other hand, using an uncalibrated sensor model results in simulated color sensor readings that differ substantially from the actual behavior. These results illustrate the importance of calibration, and show the potential for the simulation to produce both photorealistic visualizations and correct numerical 
results when the material properties of the room and the response of the sensor are carefully measured and included in the model.
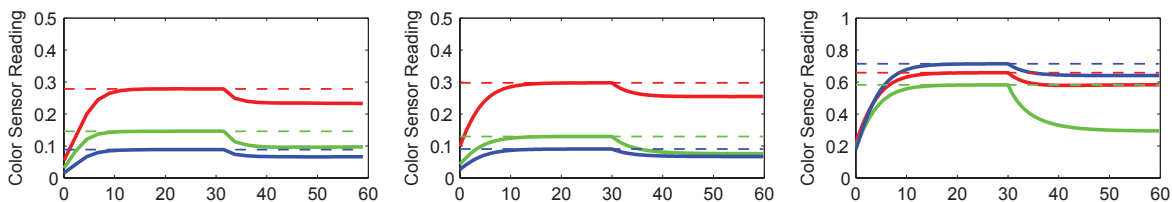

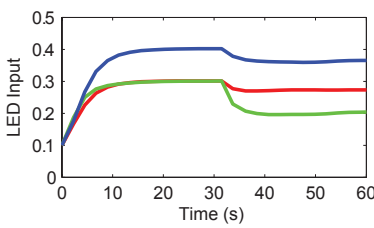

(a)

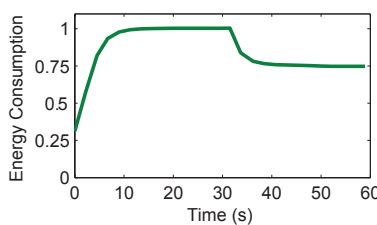

(d)

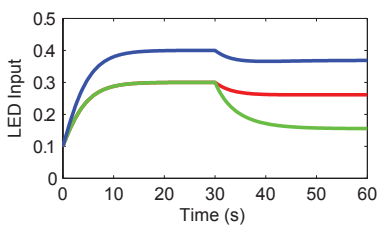

(b)

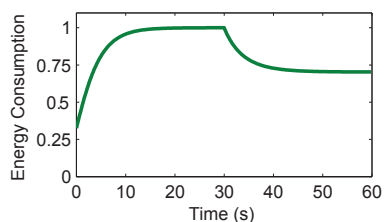

(e)

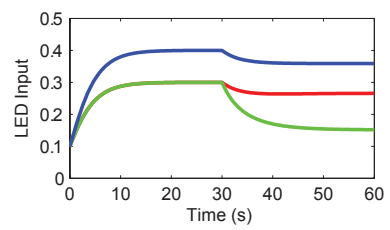

(c)

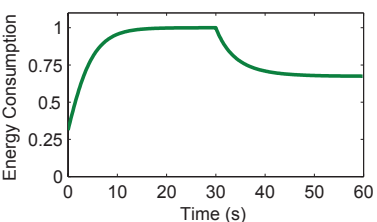

(f)

Figure 18: Color sensor readings, LED inputs (a-c) and energy consumption (d-f) for Experiment 4, including actual measurements from the physical space (left column), the simulated measurements with the calibrated color sensor model (middle column), and the results using the non-calibrated color sensor model from Experiments 1-3 (right column).

\section{Conclusions and Future Work}

We demonstrated an interactive framework for pre-visualizing and tuning the parameters of a lighting controller, based on a combination of photorealistic simulation and advanced control algorithm design. In our preliminary experiments, we showed how the simulation made it easy for a user to interactively refine the objective function for a controller in a series of design iterations. The simulation framework easily allows fixtures to be changed or moved, sensors added, or occupants introduced. We believe the tight coupling between advanced lighting control system design and environmental simulation will be productive for lighting designers and engineers, and can potentially minimize unanticipated or undesirable lighting behavior in built environments. While our experiments involved computer-generated animations of the lighting in a room over time from a single viewpoint, the camera could be easily moved as desired.

On the other hand, the experiments in the paper only represent a few scenarios out of the many possible real world environments and controller choices. More research is needed to make the simulation framework more generally applicable, such as a tool for automatically transforming a blueprint or CAD 
model into the pre-computed geometry and lightmaps required to investigate controller behavior. A bigger challenge, both technical and social, is the insertion of lighting pre-visualization tools into the typical architectural design process, especially in the early phases when the choice of lighting controller might significantly impact choices about lighting fixtures or facade elements. In future work, we plan to collaborate directly with architects and lighting designers to find ways of making the simulation framework more applicable to common practice.

Many research directions follow from this initial prototype. We are currently in the process of physically measuring light fields and transfer functions in the physical conference room under construction, to make the simulation of this space even more accurate (i.e., following on from the experiments in Section 5.4). We are also in the process of accurately characterizing and simulating new prototype sensors to be designed and deployed. We expect the lighting control simulation to inform both the development of these new sensors (e.g., necessary directional and spectral sensitivity) as well as the placement and characteristics of lighting fixtures in the constructed space.

We are also exploring several directions to make the simulation more realistic. These include moving beyond RGB sources and sensors to multispectral responses; incorporating wall-mounted sensors or sensors not collocated with fixtures; modeling occupant tracking with ceiling-mounted time-of-flight sensors, and exploring dynamic desired light fields (e.g., that adapt to moving occupants or changing weather).

A key unresolved problem, well beyond the scope of this study, is that of deciding the "right" time-varying light field for a given environment and use case. A control algorithm can be designed to accurately reach a desired setpoint, and simulation can do an excellent job of visualizing the results, but determining the setpoint itself is quite challenging. For example, the setpoint could vary according to the number, position, and pose of occupants, their activity (e.g., working in small groups, holding a discussion, watching a presentation, watching a film), and the time of day (e.g., using circadian theory to expose the occupants to warmer colors after sunset). The situation is further complicated by the challenges of designing a controller that can simultaneously satisfy the subjective preferences of multiple users, and the practical need to override the controller if the occupants find the result unsatisfactory [6]. Our continuing investigations in this area will engage lighting designers to help address these difficult questions.

\section{Acknowledgements}

This work was supported primarily by the Engineering Research Centers Program (ERC) of the National Science Foundation under NSF Cooperative Agreement No. EEC-0812056 and in part by New York State under NYSTAR contract C090145. Thanks to Brandon Andow for his perspectives on architec-

tural and lighting design, and to the anonymous reviewer for detailed suggestions on improving the presentation of the paper. 


\section{References}

[1] U.S. Energy Information Administration, Annual Energy Outlook 2013, DOE/EIA0383(2013), Washington, DC (April 2013).

[2] E. S. Lee, S. E. Selkowitz, The design and evaluation of integrated envelope and lighting control strategies for commercial buildings, Tech. rep., Lawrence Berkeley Lab., CA (United States) (1994).

[3] B. Roisin, M. Bodart, A. Deneyer, P. D'Herdt, Lighting energy savings in offices using different control systems and their real consumption, Energy and Buildings 40 (4) (2008) $514-523$.

[4] V. Singhvi, A. Krause, C. Guestrin, J. H. Garrett Jr, H. S. Matthews, Intelligent light control using sensor networks, in: Proceedings of the 3rd International Conference on Embedded Networked Sensor Systems, 2005.

[5] M. Bodart, A. De Herde, Global energy savings in offices buildings by the use of daylighting, Energy and Buildings 34 (5) (2002) 421-429.

[6] A. D. Galasiu, J. A. Veitch, Occupant preferences and satisfaction with the luminous environment and control systems in daylit offices: a literature review, Energy and Buildings 38 (7) (2006) 728-742.

[7] O. Masoso, L. Grobler, The dark side of occupants' behaviour on building energy use, Energy and Buildings 42 (2) (2010) 173-177.

[8] Y. Sheng, T. C. Yapo, C. Young, B. Cutler, A spatially augmented reality sketching interface for architectural daylighting design, IEEE Transactions on Visualization and Computer Graphics 17 (1) (2011) 38-50.

[9] M. G. Figueiro, M. S. Rea, R. G. Stevens, A. C. Rea, Daylight and productivity a possible link to circadian regulation, in: Proceedings of the Fifth International LRO Lighting Research Symposium, 2002.

[10] P. Boyce, C. Hunter, O. Howlett, The benefits of daylight through windows, Tech. rep., Lighting Research Center, Rensselaer Polytechnic Institute, Troy, NY (2003).

[11] M. S. Rea, M. G. Figueiro, A. Bierman, J. D. Bullough, Circadian light, Journal of Circadian Rhythms 8 (1) (2010) 2.

[12] E. Krietemeyer, Dynamic design framework for mediated bioresponsive building envelopes, Ph.D. thesis, Rensselaer Polytechnic Institute, Troy, NY (2013).

[13] Y. Sheng, Interactive daylight visualization in spatially augmented reality environments, Ph.D. thesis, Rensselaer Polytechnic Institute, Troy, NY (2013).

[14] J. Clarke, Energy simulation in building design, Routledge, 2012.

[15] G. W. Larson, R. Shakespeare, C. Ehrlich, J. Mardaljevic, E. Phillips, P. ApianBennewitz, Rendering with Radiance: the art and science of lighting visualization, Morgan Kaufmann San Francisco, CA, 1998.

[16] C. Reinhart, A. Fitz, Findings from a survey on the current use of daylight simulations in building design, Energy and Buildings 38 (7) (2006) 824-835.

[17] R. Cusson, J. Cardoso, Realistic Architectural Visualization with 3ds Max and mental ray, Focal Press, 2012.

[18] L. Lanier, Advanced Maya texturing and lighting, Sybex, 2006. 
[19] C. Reinhart, P. F. Breton, Experimental validation of Autodesk $® 3$ ds Max $®$ Design 2009 and Daysim 3.0, Leukos 6 (1) (2009) 7-35.

[20] K. Lagios, J. Niemasz, C. Reinhart, Animated building performance simulation (ABPS)linking Rhinoceros/Grasshopper with Radiance/Daysim, in: Proceedings of SimBuild, 2010.

[21] J. Mardaljevic, L. Heschong, E. Lee, Daylight metrics and energy savings, Lighting Research and Technology 41 (3) (2009) 261-283.

[22] C. Reinhart, J. Mardaljevic, Z. Rogers, Dynamic daylight performance metrics for sustainable building design, Leukos 3 (1) (2006) 1-25.

[23] C. Reinhart, J. Wienold, The daylighting dashboard - a simulation-based design analysis for daylit spaces, Building and Environment 46 (2) (2011) 386-396.

[24] M. Andersen, S. Kleindienst, L. Yi, J. Lee, M. Bodart, B. Cutler, An intuitive daylighting performance analysis and optimization approach, Building Research \& Information 36 (6) (2008) 593-607.

[25] S. Kleindienst, M. Andersen, Comprehensive annual daylight design through a goal-based approach, Building Research \& Information 40 (2) (2012) 154-173.

[26] S. Wang, Z. Mao, C. Zeng, H. Gong, S. Li, B. Chen, A new method of virtual reality based on Unity3D, in: International Conference on Geoinformatics, 2010.

[27] M. Zyda, From visual simulation to virtual reality to games, Computer 38 (9) (2005) $25-32$.

[28] A. Indraprastha, M. Shinozaki, The investigation on using Unity3D game engine in urban design study, ITB Journal of Information and Communication Technology 3 (1) (2009) $1-18$.

[29] L. Jia, R. J. Radke, Using time-of-flight measurements for privacy-preserving tracking in a smart room, IEEE Transactions on Industrial Informatics (In Press).

[30] F. Qureshi, D. Terzopoulos, Smart camera networks in virtual reality, Proceedings of the IEEE 96 (10) (2008) 1640-1656.

[31] M. C. Mozer, The neural network house: An environment that adapts to its inhabitants, in: Proc. AAAI Spring Symp. Intelligent Environments, 1998, pp. 110-114.

[32] S. Selkowitz, E. Lee, O. Aschehoug, Perspectives on advanced facades with dynamic glazings and integrated lightings controls, in: CISBAT 2003, Innovation in Building Envelopes and Environmental Systems, 2003.

[33] Y.-J. Wen, J. Granderson, A. M. Agogino, Towards embedded wireless-networked intelligent daylighting systems for commercial buildings, in: IEEE International Conference on Sensor Networks, Ubiquitous, and Trustworthy Computing, 2006.

[34] M. Aldrich, N. Zhao, J. Paradiso, Energy efficient control of polychromatic solid state lighting using a sensor network, in: Tenth International Conference on Solid State Lighting, 2010.

[35] B. Lee, M. Aldrich, J. A. Paradiso, Methods for measuring work surface illuminance in adaptive solid state lighting networks, in: Eleventh International Conference on Solid State Lighting, 2011.

[36] S. Afshari, S. Mishra, A. Julius, F. Lizarralde, J. T. Wen, Modeling and feedback control of color-tunable led lighting systems, in: American Control Conference (ACC), 2012, IEEE, 2012, pp. 3663-3668. 
[37] S. Afshari, S. Mishra, A. Julius, F. Lizarralde, J. D. Wason, J. T. Wen, Modeling and control of color tunable lighting systems, Energy and Buildings (In press).

[38] H. Park, J. Burke, M. B. Srivastava, Design and implementation of a wireless sensor network for intelligent light control, in: Proceedings of the 6th International Conference on Information Processing in Sensor Networks, ACM, 2007, pp. 370-379.

[39] J. Foley, A. van Dam, S. Feiner, H. J.F., Computer Graphics: Principles and Practice in C, 2nd Edition, Addison-Wesley Professional, 1995.

[40] D. Caicedo, A. Pandharipande, G. Leus, Occupancy-based illumination control of LED lighting systems, Lighting Research and Technology 43 (2) (2011) 217-234.

[41] A. Pandharipande, D. Caicedo, Daylight integrated illumination control of LED systems based on enhanced presence sensing, Energy and Buildings 43 (4) (2011) 944-950. 